\title{
The Simulation on Drilling of Carbon Fiber Reinforced Plastic Composites
}

\author{
Fei Gao, Xitian Tian \\ Institute of CAPP \& Manufacturing Engineering \\ Software \\ Northwestern Polytechnical University \\ Xi'an, China
}

\author{
Shunuan Liu, Bo Li \\ Institute of CAPP \& Manufacturing Engineering \\ Software \\ Northwestern Polytechnical University \\ Xi'an, China
}

\begin{abstract}
In order to study high speed drilling mechanism of carbon fiber reinforced plastics, a simulation on drilling is conducted by using FEM analysis software Deform-3D. The dynamic simulation of the drilling process of carbon fiber reinforced plastics is carry on basing on the elastic-plastic theory of material deformation and the thermal coupling theory, obtaining the data of drilling force and torque. The finite element model of carbon fiber reinforced plastics is established in this paper, the relevant techniques and setting principles for important parameters in the simulation are also given. The variation characteristics of drilling force and torque in the drilling process are deeply studied. The result of the research provides the support for further study of high speed drilling mechanism of carbon fiber reinforced plastics.
\end{abstract}

Keywords- Deform, simulation, CFRP, drilling force, torque

\section{INTRODUCTION}

All manuscripts must be in English. These guidelines include complete descriptions of the fonts, spacing, and related information for producing your proceedings manuscripts. Please follow them and if you have any questions, direct them to the production editor in charge of your proceedings at Conference Publishing Services (CPS): Phone +1 (714) 821-8380 or Fax +1 (714) 761-1784.

Carbon fiber reinforced plastics(CFRP) are characterized by low weight, low expansion coefficient, superior strengthto-weight and stiffness-to-weigh ratios, high fatigue strength, good thermal shock, high heat resistance and well vibration absorption. They have been used widely in airplanes, spaceships and automobile etc. [1]. But the dynamics characteristics of CFRP in each direction are different and the condition of machining is hard, the strength between the layers is low, it's easy to produce delamination and avulsion under the force of cutting while machining, and it even will be worse when drilling. CFRP is a typical difficult-tomachine composite, and the quality of machining is difficult to guarantee [2]. The study of high manganese steel with the traditional experiment method takes a long time, consumes the cutting tool materials seriously and costs us a great deal. Finite element method as an effective complement to test method can simulate the dynamic cutting process which helps to understand the physical phenomena of removing materials. It is helpful to choose the optimal tool materials, optimize the tool geometry and reduce the cost of research if the finite element method is used to study the simulation of the drilling process of CFRP.

Relatively a few studies have been developed from the last years. Arola and Ramulu [3] consider the FEM as a valid approach for the orthogonal cutting of composites, although the results from ABAQUS were different from the values obtained experimentally. Mahdi and Zang [4] proposed a quasi-static approach using the ADINA software to study the machining of composite materials, with the tool considered as rigid, the part considered as an equivalent homogeneous anisotropic material and the friction is ignored. Zitoune and Collombet [5] proposed a static model to determine the cutting forces and to examine the state of the tensile strength $\sigma$. The FEM used is an isoparametric volume type of the SAMCEF software. Calculations follow a linear static assumption and the contact is modeled by the penalties method. A 3D finite element model using the ABAQUS software was developed by Durao et al. [6] in order to study the damage of drilling operation (especially delamination) produced on CFRP plates. The model presents a mixed mode of damage allowing delamination modeling. The main objectives were to compare the influences of the drills geometry on the damage initiation and growth. Venu GopalaRao et al. [7] modeled the cutting of composite materials with the following assumptions: the tool is rigid, the fiber is isotropic and elastic, the matrix has an elastoplastic behavior and friction a value of 0.3 . To facilitate the calculations the authors have modeled the area of the workpiece closed to the tool. The fiber/matrix interface is modeled with zero thickness and detachment by a cohesive zone model (CZM). CZM represents an approach of fracture mechanics to study the effects of interface in different materials or in the same material when they are at the beginning glued together. The temperature produced between tool and work piece is neglected [2].

DEFORM is a Finite Element Method (FEM) based process simulation system designed to analyze various forming and heat treatment processes used by metal forming and related industries. The simulation of the drilling process of CFRP based on elastic - plastic theories of material deformation by the software of deform is studied in this paper, obtaining the data of drilling force and torque. 


\section{Simulating Drilling Processes of CFRP with DEFORM-3D}

Due to the number of revolutions of a drill necessary to establish characteristic behavior, drilling simulations in DEFORM are time consuming. Therefore, every effort will be made to optimize problem size.

\section{A. Set Basic Simulation Controls}

The machining type is drilling, change units to SI, the rotate speed of spindle is $4000 \mathrm{r} / \mathrm{min}$, the feed rates is $0.2 \mathrm{~mm} / \mathrm{r}$, set working environment and properties of toolworkpiece interface: define the environmental temperature as $20^{\circ} \mathrm{C}$, the shear friction factor is 0.6 and the heat transfer coefficient is $45 \mathrm{~W} /(\mathrm{m} \cdot \mathrm{K})$.

\section{B. Defining the drill geometry}

The model of twist drill can be established by using the tool module DEFORM provides, a research on conventional modeling method of the twist drill has been done. And a geometric model of twist drill is established based on the grinding principle and mathematical model of the twist drill in this paper.

Define the type of tool as twist drill, the main geometrical parameters of twist drill: the drill radius is $2.5 \mathrm{~mm}$, web thickness is $1.3 \mathrm{~mm}$, helix angle is $26^{\circ}$, point angle is $118^{\circ}$, and margin is $0.4 \mathrm{~mm}$. The material of tool is cemented carbide, and there is $3 \mu \mathrm{m}$ coating of TiN on the surface of tool.

\section{Defining the workpiece geometry}

The process of drilling is so complicated that lead to the amount of calculation very great, in order to simplify calculation process and reduce the scale of the problem, the size of the workpiece should be tried to reduce on the premise of meeting the simulation requirements of the condition. If the workpiece shape is a simple cylinder, it can be created using the geometric primitives in DEFORM. So, we will establish a simple, solid workpiece. The workpiece will be round, with a radius of $4 \mathrm{~mm}$ and a height of $2 \mathrm{~mm}$, the type of the workpiece is plastic. So the workpiece model has been established.

\section{Set Up a Finite ElementModel of CFRP}

As the material library deform $3 \mathrm{D}$ software provides do not include the model of CFRP, a new material model need to be established. When a new material model is established, some parameters of material need to be entered: constitutive equation for metal, Young's modulus, Poisson's ratio, Thermal Expansion Coefficient, Thermal Conductivity, Heat Capacity, Emissivity and so on. The constitutive relations is the most important factor for the simulation, it is a mathematicalrelation which represent mechanical properties of material. In order to acknowledgethe response of object under external force, we must know the constitutive relations material suitable for. It is difficult to describe the constitutive relation of a material accurately now, so, according to curve characteristics of the flow stress, it is an effective way to choose empirical equation as material model.
So, DEFORM software provides curve fitting techniques. Select the equation of

$$
\bar{\sigma}=\bar{\sigma}(\bar{\varepsilon}, \dot{\bar{\varepsilon}}, T)
$$

as flow stress rule in the options of flow stress, Enter a series of stress and strain data of CFRP, as the experiment was carried out at room temperature, the temperature isn't considered, so the constitutive equation chooses Rosserd model:

$$
\bar{\sigma}=c \overline{\mathcal{E}}^{n} \dot{\bar{\varepsilon}}^{m}
$$

In this equation, $\mathrm{c}, \mathrm{n}, \mathrm{m}$ are materialconstants. This model is suitable for the plastic deformation of a metal at a temperature below that at which it recrystallizes or room temperature, Fit curve by choose the option of conversion, so the fitting equation can be obtained, so does the material constitutive equation. After fitting the equation is:

$$
\bar{\sigma}=7664 \bar{\varepsilon}^{1.2} \dot{\bar{\varepsilon}}^{-0.15}
$$

Enter the parameters of Young's modulus, Poisson's ratio, Thermal Expansion, Thermal Conductivity, Heat Capacity, etc. At last, the materialmodel is stored for future reference. Thus, the finite element model of CFRP is built up.

\section{E. Mesh the Object}

Meshing is an important step in the process of creating the FEM model, there are a lot of problems raised for consideration, this requires greater workload, the form of meshing has the direct influence on the computational precision and computational scale, and the numbers of the mesh will affect the precision and scale of the result. Generally, with the increase in the numbers of the mesh come an increase in the he precision and scale of the result, so, the numbers of the mesh can be determined by correctly weighing both aspects.

In the course of cutting simulation, DEFORM will regenerate a mesh dozens or hundreds of times as the material and mesh become distorted. The new meshes are generated based on user defined parameters to keep fine elements where they are needed for resolution, and place coarse elements in other areas.

For workpiece, the grid type is absolute mesh density, and the minimum element size is determined by the feed. For a two flute drill with $0.2 \mathrm{~mm}$ feed/revolution, the feed per cutting edge is $0.1 \mathrm{~mm}$. To get two elements in the chip thickness, use a minimum element size of $0.05 \mathrm{~mm}$.

The size ratio sets the size of the largest elements, in areas where no refinement is required. For CFRP cutting simulations, set size ratio to 10 , larger size ratios may lead to substantial increases in the time required for mesh generation while the simulation is running.

The tool mesh is not as critical as the workpiece mesh. For a rigid object, the geometry is always maintained for deformation contact calculations. The mesh is only used for temperature calculations. Set grid type to relative, and set the number of elements to 20,000 . 


\section{F. Simulation Controls}

In DEFORM, the deformation is subdivided into hundreds or thousands of incremental time steps. The user defined time step gives the simulation a starting point for calculations. If it is too large, the simulation module will automatically reduce it to a more suitable value.

For drilling, we would like to have about 1 degree of rotation per time step. We can round this off to about 300 steps per revolution. The drill makes $4000 \mathrm{rev} / \mathrm{min}$, which means 1 revolution takes about $3 / 200$ of a second. (3/200) / 300 gives a time step of 0.00005 seconds per step. So set the solution steps definition to with constant time increment, and assign a value of $0.00005 \mathrm{sec}$.

It is about $3.7 \mathrm{~mm}$ from the bottom of the workpiece to the top of the drill tip. We can assume $5 \mathrm{~mm}$ of penetration is necessary for the drill to go completely through the workpiece. At $40 / 3 \mathrm{~mm} / \mathrm{sec}$, this means that $3 / 8$ seconds, or 7500 steps will be required to completely drill through this sample. Enter 7500 as the number of simulation steps. This is an estimate of the number of steps that will be calculated. Due to remeshing and automatic time step control, the actual number may be more. DEFORM will adjust for this automatically. However, a stopping control can be defined.

There are a large number of stopping controls which can be set in DEFORM. A simulation will run until it reaches one of the predefined stopping controls, or until it is stopped by the user.

We will stop when the primary tool travel (the drill) reaches $5 \mathrm{~mm}$ in the direction of travel.

Check up the data, there are no other errors or warnings, the database can be generated. After database generation is completed, exit database generation and the preprocessor.

\section{G. Define Boundary Conditions}

The environmental temperature is $20^{\circ} \mathrm{C}$, set a value of 0.6 as constant friction and set heat transfer coefficient to 40.The heat transfer convection coefficient is $0.02 \mathrm{~N} / \mathrm{sec} / \mathrm{mm} / \mathrm{C}$ and the friction type is shear friction.

\section{ANALYSIS OF THE RESULTS}

The simulation of drilling is a highly nonlinear numerical calculational problem, which requires a rigorous element in the simulation, To ensure describe the interaction of force and heat between cutting tool, workpiece and chip adequately in drilling process, the elements must be small enough to meet the requirements of non-linear numerical calculations. With the limited computing environment, great feed rate is set up to meet the requirements of meshing densities, and this will lead to cutting forces become powerful and fluctuated violently.

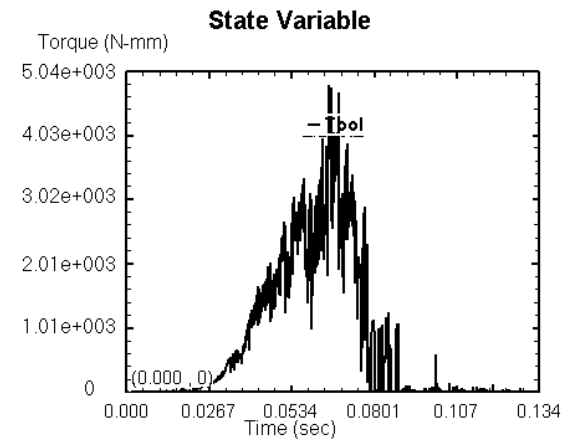

Figure 1. Drilling torque of drilling bit

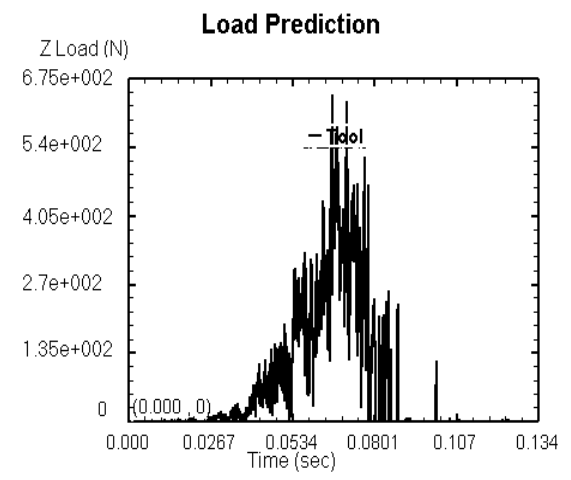

Figure 2. z-direction drilling force of drilling bit

The drilling curve is divided into three periods as shown in fig 1 and 2: in the initial period, the tool and workpiece come into contact, since the great feed engagement, the contact between drill bit and workpiece has just begun, the material hasn't been cut by cutting tool, the dramatic impact of cutting tool on the workpiece lead resulting in the beginning of the drilling force is large and growing rapidly. In the second period, the drill bit began to cut the workpiece, drilling force begin to decline, the curve tends to continue to rise after a short gentle. In the last period, with the drilling stabilize, curve become gentle but continued volatility, this is due to the small diameter of drill bit, great feed engagement and strong vibration, while the technique of AMG (Adaptive Mesh Generation) in the simulation also makes the curve volatile. With the deepening of drill bits, the strain rates vary widely in the process of drilling, the curve fluctuates greatly as a result. Also subject to the limitations of computer hardware, the chip only contains the text of a unit grid. The chin deformation and temperature characteristics cannot be represented very well, which affects the final simulation result.

\section{CONCLUSION}

(1) In this article, it is only a primary trial to apply the numerical simulation to the study of drilling

CFRP. Based on this, the temperature distribution and the influence over cutting performance of tool could be further studied during the cutting process, and also the effect of cutting parameter on cutting force and torque. 
(2) Establish the finite element model of CFRP materials, and simulate the CFRP dynamic drilling process. At last, analyze and forecast drilling force and torque of the tool in machining process.

\section{ACKNOWLEDGMENT}

The research was Sponsored by National Science Foundation of China (No.50905146)

\section{REFERENCES}

[1] Smith WF. Principles of materials science and engineering.NewYork: McGraw-Hill, 1990, PP: 699-724.

[2] D. Iliescu; D. Gehin; I. Iordanoff; F. Girot. A discrete element method for the simulation of CFRP cutting. Composites Science and Technology, 2010, PP: 73-80.
[3] Arola D; Ramulu M. Orthogonal cutting of fibre-reinforced composites: a finite element analysis. Int J Mach Tool Manuf, 1997.39(5), PP: 597-613.

[4] Mahdi M; Zhang L. A finite element model for the orthogonal cutting of fiber-reinforced composite materials. J Mater Process Technol, 2001, PP: 113-115.

[5] Zitoune R; Collombet F. Numerical prediction of the thrust force responsible of delamination during the drilling of the long-fibre composite structures.Composites A, 2007.38(3), PP: 858-866.

[6] Durao LMP; de Moura MFSF; Marques AT. Numerical simulation of the drilling process on carbon/epoxy composite laminates. Composites A, 2006.37(9), PP: 1325-1333.

[7] VenuGopalaRao G; Mahajan P; Bhatnagar N. Micro-mechanical modeling of machining of FRP composites - cutting force analysis. Compo Sci Tech, 2007.67(3-4), PP: 579-93. 